\title{
Visualization and orchestration of the dynamic molecular society in cells
}

\author{
Xuebiao Yao ${ }^{1}$, Guowei Fang ${ }^{2}$ \\ ${ }^{1}$ Division of Cellular Dynamics, Hefei National Laboratory for Physical Sciences at Nano-scale, and the University of Science \& \\ Technology of China, Hefei 230027, China; ${ }^{2}$ Department of Biological Sciences, Stanford University, Stanford, CA 94305, USA \\ Cell Research (2009) 19:152-155. doi: 10.1038/cr.2009.7; published online 20 January 2009
}

Visualization of specific molecules and their interactions in real space and time is essential to delineate how cellular plasticity and dynamics are achieved and orchestrated as perturbation of cellular plasticity and dynamics is detrimental to health. Elucidation of cellular dynamics requires molecular imaging at nanometer scale at millisecond resolution. The $1^{\text {st }}$ International Conference on Cellular Dynamics and Chemical Biology held in Hefei, China (from 12 September to 15 September, 2008) launched the quest by bringing synergism among photonics, chemistry and biology.

The conference was held in conjunction with the $50^{\text {th }}$ anniversary of the University of Science \& Technology of China (USTC). About 100 scientists and students gathered in Hefei to discuss the recent advances in molecular imaging and chemical manipulation of cell biology.

\section{Seeing is believing}

The inherent resolution limit of the light microscope has been a hurdle for visualization of molecular nanomachineries in cell biology. Jennifer

Correspondence: Xuebiao Yao ${ }^{\mathrm{a}}$, Guowei Fang ${ }^{\mathrm{b}}$

aE-mail: yaoxb@ustc.edu.cn

bE-mail: gwfang@stanford.edu
Lippincott-Schwartz (National Institutes of Health, USA) opened the meeting with a report on the latest supraresolution imaging technique named PALM (photo-activated localization microscopy) [1]. PALM enables optically imaging intracellular proteins at nanometer $(2 \sim 25 \mathrm{~nm})$ spatial resolution by isolation of single molecules at high densities (up to $\sim 10^{5} / \mu^{2}$ ) based on the serial photo-activation and subsequent bleaching of numerous sparse subsets of photo-activatable fluorescent protein molecules within a sample. By combining PALM with single-particle tracking, the same group developed a new method termed sptPALM [2], which enables the recording of several orders of magnitude more trajectories per cell than traditional single-particle tracking allows. By probing distinct subsets of molecules, sptPALM can resolve the dynamics and origins of spatial and temporal molecular heterogeneities in cells.

\section{Cell division and growth signal- ling}

During cell division, a super-protein structure called kinetochore orchestrates the equal segregation of parental genome into two daughter cells. One of the biggest challenges in the mitosis field is to illustrate the kinetochore assembly dynamics and signaling cascade underlying mitotic checkpoint. Andrea Musacchio of European Institute of Oncology, Italy, has recently succeeded in determining the crystal structure of a truncated "bonsai" version of $\mathrm{Ndc} 80$, a tetrameric complex essential for accurate spindle microtubule-chromosome attachment [3]. Xin Cai from Xuebiao Yao's laboratory (USTC, China) presented supporting evidence for the function of Ndc 80 in accurate spindlemicrotubule attachment regulated by Nek2A-mediated phosphorylation [4]. Andrea Musacchio also presented his working model accounting for the Mad2-dependent spindle checkpoint, in which he proposed that a conformational switch of Mad2 mediates its binding and inhibitory activities towards the $\mathrm{Cdc} 20$ activator of the anaphase promoting complex/cyclosome [5].

Cytoplasmic dynein is a minus-end directed microtubule-based molecular motor and plays multiple functions in mitosis. Xueliang Zhu (Shanghai Institutes for Biological Sciences, CAS, China) presented his recent finding in which NudE/Nudel (NudE-like) and Lis1 might serve as novel positive regulators of dynein in eukaryotes [6]. Both the Nudel-Lis1 and Nudeldynein interactions are crucial for dynein activity. He proposed that Nudel functions not only in dynein-mediated protein transport, but also in stabilizing kinetochore association with dynein 
to facilitate dynein-mediated chromosome poleward movement via tethering to mitosin/CENP-F. To delineate the molecular composition and interaction networks of the mammalian kinetochore, Xuebiao Yao's laboratory carried out a combination of proteomics and systemic yeast 2 -hybrid analyses. They revealed the role of Nek2A kinase in orchestrating kinetochore-microtubule attachments and mitotic checkpoint [7, 8]. Recent studies from Yao and colleagues discovered how Nuf2 tethers CENP-E for the kinetochore localization [9], and how septin 7 filaments enable the accurate translocation of CENP-E to the kinetochore [10]. Yao also presented a molecular dissection of the CENP-E interaction network in mitotic checkpoint signalling.

One central question in the cell cycle field is what controls the phase transitions? Although the accumulation of mitotic cyclins drives the transition from the $\mathrm{G} 2$ phase to the $\mathrm{M}$ phase in embryonic cells, the trigger for mitotic entry in somatic cells remains unknown. Guowei Fang (Stanford University, USA) reported his recent finding that Bora synergizes with the Aurora A kinase in controlling the G2-M transition [11]. Bora accumulates in the G2 phase and promotes Aurora A-mediated activation of Plk1, leading to the activation of cyclin-dependent kinase 1 and mitotic entry. Mechanistically, Bora interacts with Plk1 and controls the accessibility of its activation loop for phosphorylation and activation by Aurora A. While chromosome segregation in mitosis is orchestrated by protein kinase- and phosphatase-signaling cascades, early studies showed that overexpression of human phosphatase Cdc14A, an antagonist of cyclin-dependent kinase 1, affects several aspects of cell division. However, the molecular mechanism underlying $\mathrm{HsCdc} 14 \mathrm{~A}$ regulation in mitosis has remained elusive. Kai Yuan of Xuebiao Yao's laboratory showed that Cdc14A activity is regulated by an auto-inhibitory mechanism via intra- molecular interaction [12]. Plk1 interacts with and phosphorylates Cdc14A, which releases the intra-molecular interaction of Cdc14A. Using a combination of Plk1 activity reporter and a sensor to monitor Cdc14A intra-molecular interaction, Yuan is profiling the spatiotemporal dynamics of Cdc14A-Plk1 interaction in real-time chromosome segregation.

TGF- $\beta$ signalling plays pivotal roles in embryogenesis and cancer development. This signalling pathway is spatiotemporally regulated to orchestrate cellular dynamics and homeostasis. Ye-Guang Chen (Tsinghua University, China) presented studies on several newly identified proteins implicated in TGF- $\beta$ signalling [13, 14]. He showed that Dapper2 negatively regulates TGF- $\beta /$ Nodal signaling by influencing lysosomal degradation of the endocytosed TGF- $\beta /$ Nodal receptors while endofin, a member of the FYVE domain protein family, functions in endosomal trafficking. Preliminary evidence suggests that membrane lipidrafts participate in TGF- $\beta$ signalling dynamics by determining the activation of MAPK by TGF- $\beta$ [15]. Systemic acquired resistance (SAR) is a plant immune response that can be induced after a local infection. Using SAR as a model system, Xinnian Dong (Duke University, USA) showed that Arabidopsis NPR1 protein is an essential signaling component of SAR. NPR1 is sequestered in the cytoplasm as an oligomer through intermolecular disulfide bonds and S-nitrosylation of NPR1 by S-nitrosoglutathione at cysteine-156 facilitates its oligomerization, suggesting a link between pathogen-triggered redox changes and gene regulation in plant immunity [16].

\section{Structure and activity}

One of our current challenges in understanding cellular dynamics is to delineate the structural modules mediating protein-protein interactions and principles underlying the networking of such interactions. This requires an accurate understanding of their points of contact. Yunyu Shi (USTC, China) presented her studies on human PPIL1 (peptidyl prolyl isomerase-like protein 1), a component in $45 \mathrm{~S}$ U5 snRNP in activated spliceosome $\mathrm{B}^{*}$ and $35 \mathrm{~S}$ snRNP. It was believed to participate in the activation of spliceosome. Using NMR spectroscopic analysis, the solution structure of PPIL1 was determined and shown to resemble that of other members of the cyclophilin family [17]. Chemical shift perturbation experiments were employed to study the interaction between PPIL1 and SKIP (Ski-interaction protein), another essential component of $45 \mathrm{~S}$ activated spliceosome. Their studies illustrated that a novel cyclophilin-protein contact module exists in the PPIL1-SKIP complex, suggesting that protein-protein contact-mediated conformation changes may function in activation of the spliceosome.

Reversible protein tyrosine phosphorylation plays a major role in cellular signalling by changing protein's plasticity. However, delineation of the functional significance of PTPs in normal physiology and in diseases remains a major challenge in cell signaling as genetic manipulations such as gene knockout and RNAi can not resolve the involvement of a protein in two temporally separated events. Zhong-yin Zhang (Indiana University, USA) described how chemical biology approaches with potent and selective small molecule inhibitors can be used to delineate PTP function and to develop PTP-based therapeutics [e.g., 18]. Annotation of the budding yeast Saccharomyces cerevisiae genome revealed that there are 80 genes involved in oxidative stress response. Using a structural genomics approach, Congzhao Zhou and colleagues (USTC, China) revealed the structural basis of protein-mediated electron transfer in response to oxidative stress [19]. The $2 \mathrm{~b}$ proteins encoded by 
cucumovirus act as post-transcriptional gene silencing suppressors to counter host defence during infection. Adma Yuan (Singapore National University, Singapore) reported the crystal structure of $2 \mathrm{~b}$ protein complexed with a 19 bp small interfering RNA duplex [20]. The $2 \mathrm{~b}$ protein oligomerizes to form a dimer of dimers through the conserved leucine-zipper-like motif at its aminoterminal alpha-helix.

\section{Chemical biology of enzymes}

The family of 14-3-3 proteins functions as critical regulators underlying diverse signaling events in health and diseases. Given the fact that 14-3-3binding often switches the plasticity of its binding partners, Haian $\mathrm{Fu}$ (Emory University, USA) devised a high-throughput screen strategy for identifying chemicals that disrupt the association of 14-3-3 with its binding partners and carried out a large scale screening campaign [21]. Since 14-3-3 is up-regulated in certain type of lung cancers, these 14-3-3 inhibitors are of substantial significance in dissecting spatiotemporal dynamics of 14-3-3 and are also expected to be of great benefit in leading to therapeutic strategies for treating diseases such as cancer.

Polo and Aurora family kinases regulate important events during mitosis including centrosome maturation and separation, mitotic spindle assembly, and chromosome segregation. Misregulation of Aurora and Polo kinases due to genetic amplification and protein overexpression results in aneuploidy and may contribute to tumorigenesis. Therefore, chemical inhibitors of Aurora and Polo are currently undergoing phase 2 clinical trials. Jiancun Zhang (Guangzhou Institute of Biomedicine and Health, CAS, China) provided his rationale design of allosteric inhibitors for mitotic regulators such as Mps1.

Autophagy is a lysosome-dependent cellular catabolic mechanism underlying the turnover of intracellular organ- elles and long-lived proteins. Reduction of autophagy activity has been shown to lead to the accumulation of misfolded proteins in disease settings. Dawei Ma and colleagues (Shanghai Institute of Organic Chemistry, CAS, China) recently discovered some small molecule regulators of autophagy using an image-based screening strategy [22]. Interestingly, these compounds can promote degradation of long-lived proteins and reduce the levels of expanded polyglutamine repeats in cultured cells, raising the possibility that some of these drugs may be useful for the treatment of human diseases associated with the accumulation of misfolded proteins.

During cell division, chromosome segregation is orchestrated by the interaction of spindle microtubules with the centromere. A dramatic remodeling of interpolar microtubules into an organized central spindle between the separating chromatids is required for the initiation and execution of cytokinesis. It becomes increasingly clear that a cohort of kinesin molecules cooperate to orchestrate the spindle dynamics in mitosis. To dissect the spatiotemporal dynamics, Xuebiao Yao and colleagues set up a combination of phenotype-based screen for mis-aligned chromosomes in mitosis and inhibition of CENP-E activity in vitro. Treatment of these novel CENP-E inhibitors resulted in chromosome alignment errors, which is reminiscent of siRNA-mediated knockdown of CENP-E. The excitement ahead is to use these valuable chemical tools to dissect the temporal function of CENP-E in mitosis.

Kaixian Chen (Shanghai Institutes for Biological Sciences, CAS, China) exemplified the case study of application of chemical biology strategies for pharmacological lead identification and development of traditional Chinese medicine. He elaborated how molecular dynamics and network information can be used for intervening disease development and progression. Given the differential involvement of certain key molecules in health and diseases, he stressed the importance of allosteric modulators in harnessing disease-associated target activity.

\section{Cellular plasticity and manipula- tion}

Glioblastomas display a hierarchy of neoplastic cells with a restricted population of cells that exhibit extensive self-renewal and the capacity to generate complex tumors that recapitulate parental tumours upon xenotransplantation. Xiao-Fan Wang (Duke University, USA) presented an unexpected excitement in which $\mathrm{N}$-myc downstream-regulated gene 4 ( $\mathrm{Ndrg} 4)$ is critical for the survival of glioblastoma cells. Knockdown of Ndrg4 induces an increase in apoptosis, indicating that Ndrg4 diverges in function from its closely related tumour suppressive family members in the context of glioblastoma.

Cell-based phenotypic and pathwayspecific screens of synthetic small molecules and natural products have historically provided useful chemical ligands to modulate and/or study complex cellular processes. Sheng Ding and colleagues (Scripps Research Institute, USA) discovered small molecules that can be used to selectively regulate stem cell fate and developmental signalling pathways [23, 24]. Such molecules will provide useful tools to dissect stem cell plasticity, and may ultimately contribute to regenerative medicine for tissue repair. Reverberatory activity within neuronal cell assembly is thought to represent the online memory trace in the brain and to serve as an elementary unit in thought process. Using cultured hippocampal neurons as a model system, Guoqiang Bi and colleagues (USTC, China) revealed that activityinduced synaptic plasticity could drive the emergence of reverberatory activity in small networks [25]. It would be of great interest to illuminate the molecular regulatory network underlying the reverberatory activity. 


\section{Perspectives}

Accurate determination of the spatiotemporal dynamics of interacting proteins within a cell is essential for understanding their biological function. Recent success in the use of two-color PALM further demonstrates the feasibility to directly visualize molecular interactions within cellular structures at the nanometer scale [26]. Given the fact that much of the excitement in the genome-wide analyses of protein-protein interaction networks was recently constructed based on biochemical and computational analyses in different organisms [e.g., 27], one obvious question is to visualize how a "party hub" protein like BRCA1 interacts with multiple partners simultaneously, and whether perturbation of the "party hub" would change the cellular plasticity leading to diseases. In addition, it would be of great interest to examine whether finetuning of the spatiotemporal dynamics of specific protein-protein interactions in disease settings is sufficient for the cure. In both cases, small molecules targeting protein-protein interactions will be extremely valuable for illuminating the protein-protein interaction network orchestration in health and correcting aberrant protein-protein interactions in diseases. There is no doubt that consolidation of protein-protein interaction network and circuitry combined with nano-scale illumination of molecular dynamics will enable us to delineate the molecular physiology and pathogenesis of diseases.

\section{References}

1 Betzig E, Patterson GH, Sougrat R, et al. Imaging intracellular fluorescent proteins at nanometer resolution. Science 2006; 313:1642-1645.

2 Manley S, Gillette JM, Patterson GH, et al. High-density mapping of single-molecule trajectories with photoactivated localization microscopy. Nat Methods 2008; 5:155-157.

3 Ciferri C, Pasqualato S, Screpanti E, et al. Implications for kinetochore-microtubule attachment from the structure of an engineered Ndc80 complex. Cell 2008; 133:427-439.

4 Du J, Cai X, Yao J, et al. The mitotic checkpoint kinase NEK2A regulates kinetochore microtubule attachment stability. Oncogene 2008; 27:4107-4114.

5 Mapelli M, Massimiliano L, Santaguida S, Musacchio A. The Mad2 conformational dimer: structure and implications for the spindle assembly checkpoint. Cell 2007; 131:730-743.

6 Liang Y, Yu W, Li Y, et al. Nudel modulates kinetochore association and function of cytoplasmic dynein in $\mathrm{M}$ phase. Mol Biol Cell 2007; 18:2656-2666.

7 Lou Y, Yao J, Zereshki A, et al. NEK2A interacts with MAD1 and possibly functions as a novel integrator of the spindle checkpoint signaling. J Biol Chem 2004; 279:20049-20057.

8 Fu G, Ding X, Yuan K, et al. Phosphorylation of Sgo1 by NEK2A is essential for chromosome segregation. Cell Res 2007; 17:608-618.

9 Liu D, Ding X, Du J, et al. Nuf2 interacts with CENP-E and is essential for a stable spindle microtubule-kinetochore attachment. J Biol Chem 2007; 282:2141521424.

10 Zhu M, Wang F, Yan F, et al. Septin 7 interacts with CENP-E and is required for its kinetochore localization. $J$ Biol Chem 2008; 283:18916-18925.

11 Seki A, Coppinger JA, Jang CY, Yates JR, Fang G. Bora and the kinase Aurora a cooperatively activate the kinase Plk1 and control mitotic entry. Science 2008 320:1655-1658.

12 Yuan K, Hu H, Guo Z, et al. Phosphoregulation of $\mathrm{HsCDC} 14 \mathrm{~A}$ by polo-like kinase 1 is essential for mitotic progression. J Biol Chem 2007; 282:2741427423.

$13 \mathrm{Su}$ Y, Zhang L, Gao X et al. The evolutionally conserved activity of Dapper2 in antagonizing TGF-beta signaling. FASEB J 2007; 21:682-690.

14 Chen YG, Wang Z, Ma J, Zhang L, Lu Z. Endofin, a FYVE domain protein, interacts with Smad4 and facilitates TGF-beta signaling. J Biol Chem 2007; 282:9688-9695.

15 Wei Zuo and Ye-Guang Chen. Specific activation of MAPK by TGF- $\beta$ receptors in lipid rafts is required for epithelial cell plasticity. Mol Biol Cell 2008 Dec 3. doi: 10.1091/mbc.E08-08-0852
16 Tada Y, Spoel SH, Pajerpwska-Mukhtar $\mathrm{K}$, et al. Plant immunity requires conformational charges of NPR1 via Snitrosylation and thioredoxins. Science 2008; 321:952-956.

$17 \mathrm{Xu} \mathrm{C}$, Zhang J, Huang X, et al. Solution structure of human peptidyl prolyl isomerase-like protein 1 and insights into its interaction with SKIP. $J$ Biol Chem 2006; 281:15900-15908.

18 Boutselis IG, Yu X, Zhang ZY, Borch RF. Synthesis and cell-based activity of a potent and selective protein tyrosine phosphatase 1B inhibitor prodrug. JMed Chem 2007; 50:856-864.

19 Yu J, Zhang NN, Yin PD, Cui PX, Zhou CZ. Glutathionylation-triggered conformational changes of glutaredoxin Grx 1 from the yeast Saccharomyces cerevisiae. Proteins 2008; 72:1077-1083.

20 Chen HY, Yang J, Lin C, Yuan YA. Structural basis for RNA-silencing suppression by Tomato aspermy virus protein 2b. EMBO Rep 2008; 9:754760.

21 Li Z, Zhao L, Park HR, et al. Downregulation of 14-3-3zeta suppresses anchorage-independent growth of lung cancer cells through anoikis activation. Proc Natl Acad Sci USA 2008; 105:162167.

22 Zhang L, Yu J, Pan H, et al. Small molecule regulators of autophagy identified by an image-based high-throughput screen. Proc Natl Acad Sci USA 2007; 104:19023-19028.

23 Chen S, Do JT, Zhang Q, et al. Selfrenewal of embryonic stem cells by a small molecule. Proc Natl Acad Sci USA 2006; 103:17266-17271.

$24 \mathrm{Xu}$ Y, Shi Y, Ding S. A chemical approach to stem-cell biology and regenerative medicine. Nature 2008; 453:338344.

25 Lau PM, Bi GQ. Synaptic mechanisms of persistent reverberatory activity in neuronal networks. Proc Natl Acad Sci USA 2005; 102:10333-10338.

26 Han JD, Bertin N, Goldberg DS, et al. Evidence for dynamically organized modularity in the yeast protein-protein interaction network. Nature 2004; 430:88-93.

27 Shoff H, Galbraith CG, Galbraith JA, et al. Dual-color superresolution imaging of genetically expressed probes within individual adhesion complexes. Proc Natl Acad Sci USA 2007; 104:2030820313 